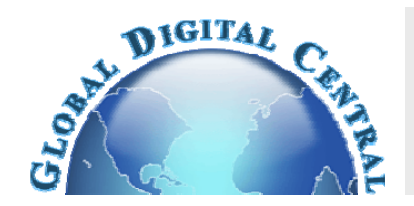

\author{
Frontiers in Heat and Mass Transfer \\ Available at www.ThermalFluidsCentral.org
}

\title{
MHD BOUNDARY LAYER FLOW AND HEAT TRANSFER OF A NANOFLUID PAST A RADIATIVE AND IMPULSIVE VERTICAL PLATE
}

\author{
G. Dharmaiah ${ }^{\mathrm{a}, \dagger}$, N. Vedavathi ${ }^{\mathrm{b}}$, CH. Baby Rani ${ }^{\mathrm{c}}$, and K.S. Balamurugan ${ }^{\mathrm{d}}$ \\ ${ }^{a}$ Department of Mathematics, Narasaraopeta Engineering College, Yellamanda, Narsaraopet, Andhra Pradesh, 522601, India. \\ ${ }^{b}$ Department of Mathematics, Koneru Lakshmaiah Education Foundation, Vaddeswaram, Guntur, Andhra Pradesh, 522502, India. \\ ${ }^{c}$ Department of Mathematics, V. R. Siddhartha Engineering College, Kanuru, Andhra Pradesh, 520007, India. \\ ${ }^{d}$ Department of Mathematics, RVR \& JC College of Engineering, Chowdavaram, Guntur, Andhra Pradesh, 522019, India.
}

\begin{abstract}
The time-dependent flow past an impulsively started vertical infinite plate in a viscous electrically conducting natural convective incompressible Nano-fluid is considered in this article by taking into account the effects of heat absorption, heat generation and radiation. An analytical study is performed to obtain exact solutions for water-based Nano-fluid $\mathrm{TiO}_{2}$. The dimensionless governing equations for this investigation are solved analytically by using the small perturbation Technique. The effects of various physical parameters on velocity, temperature fields are presented graphically. With the aid of these, the expression for the skin-friction and Nusselt number profiles was done with the help of tables.
\end{abstract}

Keywords: Heat Transfer, Nano-fluid, Impulsive Plate, $M H D$.

\section{INTRODUCTION}

Nanofluids are suspensions of nanoparticles in base liquids, another test for thermal sciences gave by nanotechnology. Nanofluids have exceptional highlights not the same as regular strong fluid blends in which $\mathrm{mm}$ or $\mu \mathrm{m}$ estimated particles of metals and non-metals are scattered. Because of their incredible qualities, nanofluids find wide applications in improving heat transfer. Research work away at the idea, heat transfer upgrade instrument, and utilization of the nanofluids is still in its essential stage. The blend of suspended nanoparticles in a base fluid is generally alluded to as a nanofluid. Nature is loaded with nanofluids, similar to blood, a complex organic nanofluid where diverse nanoparticles (at sub-atomic level) achieve distinctive capacities, and useful segments effectively react to their nearby condition. As indicated by the sorts of fluids (natural and inorganic) and sorts of nanoparticles, one can get diverse kinds of nanofluids like process extraction nanofluids, ecological (contamination controlling nanofluids), bio and pharmaceutical nanofluids. Another class of polymer nanofluids, draglessening nanofluids, go for improved warmth exchange, and in addition, stream erosion decrease. An extensive variety of dynamic selfget together instruments for nanoscale structures begin from a suspension of nanoparticles in liquid. Present day nanotechnology enables one to process and deliver materials with normal crystallite measure $<50 \mathrm{~nm}$. Nanofluid have some one of a kind highlights that are very not quite the same as ordinary two-stage stream blends in which $\mu \mathrm{m}$ as well as $\mathrm{mm}$ particles are suspended. Contrasted with an ordinary fluid and regular two-stage blend, the nanofluid has higher warm conductivity, does not square stream channels, and initiates a little weight drop. Strong particles are included as they lead warm much superior to a fluid. What's more, nanoparticles oppose sedimentation, when contrasted with bigger particles, because of Brownian movement and interparticle powers and have substantially higher surface territory (1,000-time) which upgrades the thermal conduction of nanofluids since heat transfer happens on the surface of the liquid. Three properties that make nanofluids promising coolants are: (i) expanded warm conductivity,(ii) expanded single-stage warm exchange, and (iii) expanded basic warmth motion. Research has demonstrated that generally little measures of nanoparticles, of the request of 5 vol.per penny or less, can upgrade warm conductivity of the base liquid to a vast degree.

In this way, abusing the extraordinary attributes of nanoparticles, nanofluids are made with two highlights essential for warm exchange systems:(i) outrageous steadiness, and (ii) ultra-high warm conductivity. Choi et al.,(2001) first arranged nanofluids by blending nano particles with liquids. From that point forward, there has been quick advancement in the systhesis procedures for nanofluids. Notwithstanding, there isn't yet a standard planning technique for nanofluids. Distinctive investigations arranged nanofluids utilizing diverse methodologies. There are two principal techniques to acquire nanofluids: (a) two-advance process in which nanoparticles are first delivered as a dry powder, commonly by a latent gas. The subsequent nanoparticles are then scattered into a liquid. This strategy may bring about an expansive level of nanoparticle agglomeration.(b) substance approach utilizing wet innovation, a solitary advance approach, is rising as an intense technique for developing nanostructures of various metals, semiconductors, non-metals, and cross breed frameworks. Focal points offered by nanochemistry are that surface functionised nanoparticles and nanorods of metals or inorganicsemiconductors, dispersible in wide assortment of media, for example, water, can be promptly arranged with exact control to create monodispersed nanostructures.

Under the estimation of a generally monodisperse suspension, the diffusing cross area can be identified with the result of the molecule frame factor, which is controlled by the state of the molecule, and the structure factor for relationships between's various particles. Various properties of the suspensions, for example, normal molecule measure, net attributes of shape (e.g. round, or elliptical, proportion of surface zone to volume, thickness of the center and the shells of the particles),

† Corresponding author. dharma.g2007@gmail.com 
the polydispersity and the degree to which particles stay confined or have agglomerated can be acquired decently effortlessly.

Conceivable instruments considered for this expansion are nanoparticle dissemination and limit layer diminishing, scattering and improved turbulence. Increment in basic warmth motion might be credited to modification of nucleation site by nanoparticle. Various considers have exhibited peculiar improvement of warm conductivity when little measure of nanoparticles or nanofibres of oxides $\left(\mathrm{Al}_{2} \mathrm{O}_{3}\right.$, $\mathrm{ZrO}_{2}, \mathrm{SiO}_{2}, \mathrm{CuO}$ ), metals ( $\mathrm{Au}, \mathrm{Ag}, \mathrm{Cu}$ ), Carbon (PyC, precious stone, C60) are suspended in like manner liquids (water, ethylene glycol, oils, and so forth). Warm conductivity has gotten the most consideration, however a few gatherings have as of late started investigations of other warmth exchange properties. Hardly any examinations have been directed on the rheology of nanofluids, despite the fact that the thickness of a nanofluid is as essential as thermal conductivity with regards to useful applications. High thermal conductivity and extraordinary steadiness have dependably been wanted for warm exchange liquids with particles. Liquids having this vital blend of highlights did not exist till the approach of nanofluids. Nanofluid innovation could make the procedure more vitality productive and savvy. These nanofluids could be utilized as a part of an extensive variety of modern applications. Interest for ultra-elite cooling in hardware has been expanding, and traditional upgraded surface methods have achieved their point of confinement as to enhancing heat exchange. Since nanoparticles are moderately substantially littler than the measurement of microchannel stream sections, smooth-streaming nanofluids could give the arrangement. Since nanofluids can stream in microchannels without stopping up, they would be reasonable coolants. These could upgrade cooling of microelectro mechanical frameworks (MEMS) under extraordinary warmth motion conditions. Motor coolants (ethylene glycol/water blends), motor oils, programmed transmission liquids, and other engineered high-temperature warm exchange liquids as of now have characteristically poor warmth exchange abilities, they could profit by the high warm conductivity offered by nanofluids. Nanofluids could be utilized as metalworking coolant liquids for pounding and cleaning parts. Sun based vitality frameworks could exploit nanofluids to improve warm exchange from sun powered authorities to capacity tanks. Nanofluids could enhance the warmth exchange capacities of current mechanical HVAC and refrigeration frameworks. Nanofluids could likewise be intended for properties other than modern warmth exchange. For instance, the biomedical field could scatter attractive nanoparticles into blood, manage these attractively to a dangerous tumor, and after that utilization a laser or attractive field that exchanges vitality to the particles to wreck the tumor without altogether warming the blood or harming solid tissue adjacent. Directed nearby conveyance of medications or radiation would likewise be conceivable utilizing attractively guided nanoparticles in the circulation system. It is well known that the convectional heat transfer fluids such as water, mineral oil and ethylene glycol have poor heat transfer properties compared to those of most solids in general. Increasing the coefficient of heat transfer is important for all industrial applications. Ultra-highperformance can be obtained by suspending ultrafine solid particles in a convectional fluid. The thermal conductivity of solid metals is larger than fluids; therefore suspending these particles can increase thermal conductivity and heat transfer performance. Based on different scientific and practical applications in different industrial areas, the study of two-dimensional boundary layer flow, heat and mass transfer over a porous surface is quite important.

Heat transfer analysis of flow over a porous surface has received much practical interest with abundant applications. Specifically, heattreated materials traveling between a feed roll and wind-up roll or materials manufactured by extrusion, glass-fiber and paper production, cooling of metallic sheets or electronic chips, crystal growing just to name a few. In these cases, the final desired characteristics depend on the rate of cooling in the process.
The broad range of current and future applications involving nanofluids motivated the researchers to find effective techniques for enhancing the heat transfer (Akbarzadeh et al. 2016; Wang and Mujumdar 2008; Hamad, 2011). Recently, the researchers [Raju et al. (2016), Arpaci (1987); Raju et al. (2016); Sandeep et al. (2016); Woods(1975); Babu et al.(2016).] studied the heat and mass transfer of MHD flows through different channels. Buongiorno (2006) analyzed the flow characteristics of viscous, with incompressible fluids with suspended nano-sized solid particles high significant due to the application of such fluids in heat transfer devices. Xie et al. (2010) have examined magnesium oxide nanofluids; higher thermal conductivity and lower viscosity with ethylene glycol-based nanofluids containing oxide nanoparticles. Electro-oxidation of ethylene glycol has attracted considerable interest for mobile, stationary and portable applications owing to its high theoretical energy capacity, high boiling point and high efficiency of electric power conversion has been analyzed in a review article by Yue et al. (2012). Satya Narayana and Venkateswarlu (2016) examined heat transfer analysis of water-based nanofluid over a stretching sheet using different types of nanoparticles such as $\mathrm{Cu}, \mathrm{Ag}$, $\mathrm{TiO}_{2}$, and $\mathrm{Al}_{2} \mathrm{O}_{3}$. One of the problems by Zhang (2017 \& 2018) of these membranes is their high flow resistances because of nanoscale confining pores, which can result in fairly small fluxes of the membranes. It was shown that this slow flow is due to the nanoscale pore radius, the increased effective viscosity of the liquid in the confining pores and the significant non-continuum effect of the liquid across the pore radius (Zhang, 2016). In present situation authors are very much interested doing research in nanofluids, as they can be found in computers, games consoles, mobile phones, blue-ray players, TVs as well as cars, planes and medical technology likewise the authors Njane and Makinde (2013) are studied the effect of magnetic field on boundary layer flow of incompressible electrically conducting water based nanofluids and a variation of heated vertical slip boundary condition. The authors (Nasrin et al., 2012) analysed heat transfer and fluid flow of natural convection with $\mathrm{Al}_{2} \mathrm{O}_{3}$ - water nanofluid studied in a vertical closed chamber. Here the authors used numerical method with finite element technique with Galerkin's weighted residual simulation. They examined the parameters such as nanoparticle volume fraction, prandtl number and cavity aspect ratio are due to the presence of nanoparticle the heat transfer effects are identified and based on this effects the results are pointed as, by decreasing the prandlt number amplifying the effects of nanoparticles due to increased effective thermal diffusivity and a correlation is matured graphically for the average nusselt number as a function of the cavity aspect ratio. Bejan (1982) described the conceptual design of heat transfer associated with some of the most fundamental convective processes.

Ibrahim and Makinde (2015) have been examined the effect of slip and convective boundary condition on magnetohydrodynamic (MHD) stagnation point flow and heat transfer due to Casson nanofluid. Makhatar et al., (2015) analyzed the flow reversal of the fully developed flow mixed convection of nanofluids in a vertical channel filled with porous medium. Makinde (2005) discussed the combined heat transfer effect on free convectional flow due to vertical porous plate in the presence of magnetic field. Ramesh et al., (2016) investigated the mathematical modeling for boundary layer flow and heat transfer past an inclined stationary/moving flat plate with a convective boundary condition.

Motivated by some of the researchers mentioned above and its applications in various fields of science and technology, it is of interest to discuss and analyze the heat source and radiation effects on the free convection heat and mass transfer flow of nanofluid over a vertical plate embedded in a porous medium in the presence of impulsive boundary conditions. Majority of the studies, reported in the literature, on free convective heat and mass transfer in nanofluid embedded in a porous medium deal with local similarity solutions and nonsimilarity solutions. But in the present study, the governing equations are solved using similarity transformations. 


\section{ANALYSIS OF THE FLOW OF THE PROBLEM}

This paper considers the time-dependent MHD free convection evolution of nanofluid boundary layer flow and thermal process taking place over a flat plate is moving vertically in the presence of uniform transverse magnetic field of strength $\mathrm{B}_{0}$. The surface is further subjected to a normally applied radiative heat flux with strength $\mathrm{qr}_{\mathrm{r}}$. For simplicity, infinite plate is taken, possessing only the velocity component $\mathrm{u}(\mathrm{y}, \mathrm{t})$ acting along the $\mathrm{x}$-axis with $\mathrm{y}$ representing the normal coordinate to it. The initial motional less fluid is surrounded by a constant ambient temperature $\mathrm{T}_{\infty}$. taking the water as the base fluid, the considered nanofluid is composed of nanoparticle $\mathrm{TiO}_{2}$ whose thermophysical to be used in the nanofluid model.

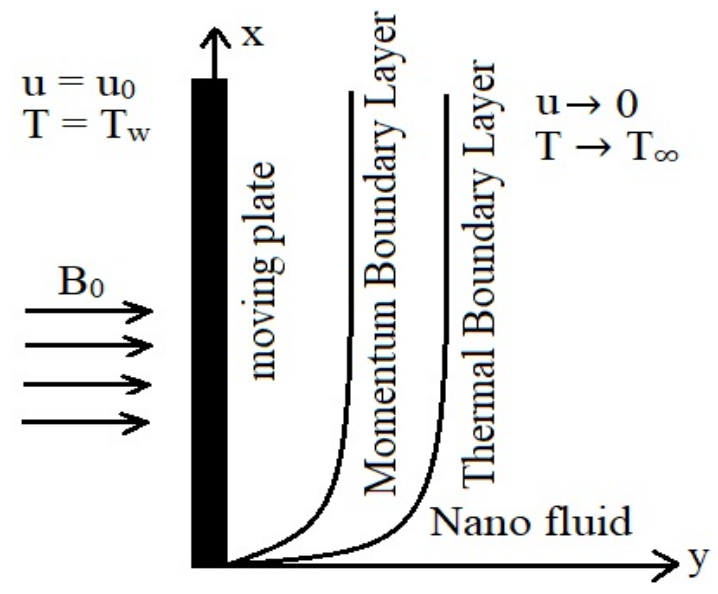

Fig. 1 Geometry of the Problem

In view of these approximations, the momentum and thermal equations are

$$
\begin{aligned}
& \frac{\partial u}{\partial t^{*}}=\frac{1}{\rho_{n f}}\left[\mu_{n f} \frac{\partial^{2} u}{\partial y^{*^{2}}}+(\rho \beta)_{n f} g\left(T-T_{\infty}\right)-\sigma u B_{0}^{2}\right] \\
& \frac{\partial T}{\partial t^{*}}=\frac{1}{\left(\rho C_{p}\right)_{n f}}\left[k_{n f} \frac{\partial^{2} T}{\partial y^{* 2}}-\frac{\partial q_{r}}{\partial y^{*}}-Q_{0}\left(T-T_{\infty}\right)\right]
\end{aligned}
$$

The boundary conditions for the problem are given by

$$
\begin{array}{ll}
u=0, T=T_{\infty} \quad \text { for any } y \geq 0 \quad(t \leq 0) \\
u=u_{0}, T=T_{w} \quad \text { at } y=0 \quad(t>0) \\
u \rightarrow 0, T \rightarrow T_{\infty}, \quad \text { as } y \rightarrow \infty
\end{array}
$$

Refer nomenclature for the variables and quantities.

For nanofluids the expressions for $\mu_{n f}, \rho_{n f},(\rho \beta)_{n f}$, and $\left(\rho C_{p}\right)_{n f}$ are given by (Tiwari and Das 2007)

$\mu_{n f}=\frac{\mu_{f}}{(1-\phi)^{2.5}}$,

$\rho_{\text {nf }}=(1-\phi) \rho_{f}+\phi \rho_{s}$,

$(\rho \beta)_{n f}=(1-\phi)(\rho \beta)_{f}+\phi(\rho \beta)_{s}$,

$\left(\rho C_{p}\right)_{n f}=(1-\phi)\left(\rho C_{p}\right)_{f}+\phi\left(\rho C_{p}\right)_{s}$

The nanoparticles of spherical shape are accounted for in Eq. (4).

The Hamilton and Crosser thermal conductivity model is adopted (kakac and Pramunjaroenkij 2009)

$k_{n f}=k_{f}\left(\frac{k_{s}+2 k_{f}-2 \phi\left(k_{f}-k_{s}\right)}{k_{s}+2 k_{f}+2 \phi\left(k_{f}-k_{s}\right)}\right)$
Following Magyari and Pantokratoras (2011), the Rossenland approximation is adopted for radiative flux $\mathrm{q}_{\mathrm{r}}$, namely

$q_{r}=-\frac{4 \sigma^{*}}{3 k^{*}} \frac{\partial T^{4}}{\partial y^{*}}$

Table 1 Thermo-physical properties (Turkyilmazoglu 2013)

\begin{tabular}{|c|c|c|}
\hline $\begin{array}{c}\text { PHYSICAL } \\
\text { PROPERTIES }\end{array}$ & WATER & $\begin{array}{c}\text { TITANIUM } \\
\text { OXIDE(TIO } 2)\end{array}$ \\
\hline $\mathrm{C}_{\mathrm{P}}(\mathrm{J} / \mathrm{KGK})$ & 4179 & 686.2 \\
$\mathrm{P}\left(\mathrm{KG} / \mathrm{M}^{3}\right)$ & 997.1 & 4250 \\
$\mathrm{~K}(\mathrm{~W} / \mathrm{MK})$ & 0.613 & 8.9538 \\
$\mathrm{BX} 10^{-5}(1 / \mathrm{K})$ & 21 & 0.9 \\
\hline
\end{tabular}

Assuming small temperature difference, it is possible to approximate $T^{4} \cong 4 T_{\infty}^{3} T-3 T_{\infty}^{4}$

Let us introduce the following dimensionless variables:

$t=\frac{u_{0}^{2} t^{*}}{v_{f}}, y=\frac{y^{*} u_{0}}{v_{f}}, U=\frac{u}{u_{0}}, \theta=\frac{T-T_{\infty}}{T_{W}-T_{\infty}}$

Here $u_{0}=\left[g \beta_{f}\left(T_{w}-T_{\infty}\right) v_{f}\right]^{\frac{1}{3}}$ is the Characteristic velocity, $\operatorname{Pr}=\frac{v_{f}}{k_{f}}$ is the Prandtl number, $N r=\frac{16 \sigma^{*} T_{\infty}^{3}}{3 k_{f} k^{*}}$ is the radiation parameter, $\quad M=\frac{v_{f} \sigma B_{0}^{2}}{u_{0}^{2} \rho_{f}}$; and $Q=\frac{Q_{0} v_{f}}{u_{0}^{2} \rho_{f} C_{p}}$ is the heat source parameter.

In Eq. (1) \& (2) with the boundary conditions (3), we get

$D \frac{\partial U}{\partial t}=F \frac{\partial^{2} U}{\partial y^{2}}+E G r \theta-M U$

$A \frac{\partial \theta}{\partial t}=\frac{B}{\operatorname{Pr}} \frac{\partial^{2} \theta}{\partial y^{2}}-C \theta$

With the boundary conditions

$$
\begin{aligned}
t \leq 0: U & =0, \theta=0 \\
t>0: U & =1, \theta=1 \quad \text { at } y=0 \\
U & \rightarrow 0, \theta \rightarrow 0 \quad \text { as } y \rightarrow \infty
\end{aligned}
$$

\section{NUMERICAL SOLUTION}

Eqs. (8) \& (9) are coupled non-linear partial differential equations whose solutions in closed form are difficult to obtain. To solve these equations by converting into ordinary differential equations, the unsteady flow is superimposed on the mean steady flow, so that in the neighborhood of the plate, the expressions for velocity and temperature are assumed as:

$\mathrm{U}(\mathrm{y}, \mathrm{t})=\mathrm{u}_{0}(\mathrm{y})+\varepsilon \mathrm{e}^{\delta \mathrm{t}} \mathrm{u}_{1}(\mathrm{y})+\mathrm{O}\left(\varepsilon^{2}\right)$

$\theta(\mathrm{y}, \mathrm{t})=\theta_{0}(\mathrm{y})+\varepsilon \mathrm{e}^{\delta \mathrm{t}} \theta_{1}(\mathrm{y})+\mathrm{O}\left(\varepsilon^{2}\right)$

where $\varepsilon<<1$ is a parameter.

Eq.s (8) and (9) are reduced to

$$
\begin{aligned}
& F u_{0} "-M u_{0}=-E G r \theta_{0} \\
& F u_{1}{ }^{\prime \prime}-M u_{1}-D t u_{1}=-E G r \theta_{1} \\
& \theta_{0} "-\frac{\operatorname{Pr} C}{B} \theta_{0}=0
\end{aligned}
$$


$\theta_{1}^{\prime \prime}-\left(\frac{\operatorname{Pr}(C+A t)}{B}\right) \theta_{1}=0$

The boundary conditions (10) becomes

$u_{0}=1, u_{1}=0, \theta_{0}=1, \theta_{1}=1$ at $y=0$

$u_{0}=0, u_{1}=0, \theta_{0}=0, \theta_{1}=0$ as $y \rightarrow \infty$

By solving Eqs. (13) to (16) using the boundary conditions (17), we get

$U(y, t)=\left(p_{4} e^{-\lambda_{3} y}+p_{3} p_{2} G r e^{-\lambda_{1} y}\right)+\varepsilon e^{\delta t}\left(p_{7} e^{-\lambda_{4} y}+p_{6} p_{2} e^{-\lambda_{2} y}\right)$

$\theta(y, t)=e^{-\lambda_{1} y}+\varepsilon e^{\delta t} e^{-\lambda_{2} y}$

The shearing stress at the plate in dimensional form is given by

$\tau=\left(\frac{\partial U}{\partial y}\right)_{y=0}=\left(-\lambda_{3} p_{4}-\lambda_{1} p_{3} p_{2}\right)+\varepsilon e^{\delta t}\left(-\lambda_{4} p_{7}-\lambda_{2} p_{6} p_{2} G r\right)$

Similarly, the rate of heat transfer at the plate/Nusselt number is given by

$N u=-\left(\frac{\partial \theta}{\partial y}\right)_{y=0}=\lambda_{1}+\varepsilon e^{\delta t} \lambda_{2}$

\section{RESULTS AND DISCUSSIONS}

In order to bring out the salient features of the flow, heat transfer characteristics with nanoparticles, the results are presented in Figs. 2- 9 and in Tables 2 and 3. The effects of nanoparticles on the velocity and the temperature distributions as well as on the skin friction and the rate of heat transfer coefficients are discussed numerically. We have chosen here $\varepsilon=0.01, \mathrm{t}=1, \mathrm{Q}=0.1, \mathrm{Pr}=6.785, \delta=0.1, \mathrm{Nr}=1, \mathrm{M}=1, \mathrm{Gr}=1$, $\phi=0.10$. We consider $\mathrm{TiO}_{2}$ with water as the base fluid. Table 1 shows the thermo-physical properties of water and the element $\mathrm{TiO}_{2}$.

The effect of Grashof number Gr on velocity is shown in Figure 2. It is observed that the velocity increases with the increasing values of Gr. Physically, this is possible because as the Grashof number increases, the contribution from the thermal buoyancy near the plate significant and hence a rise in the velocity near the plate is observed. This gives rise to an increase in the induced flow. For higher values of Gr, the fluid velocity increases the plate velocity in the regions close to the boundary. For different values of magnetic field parameter $M$, the profiles of velocity is plotted in Fig. 3. It is obvious that the velocity distribution across the boundary layer decreases, as the magnetic field parameter increases. The velocity profiles $u(y, t)$ in case of radiation is shown in Figure 4. It is revealed from this figure that radiation parameter $\mathrm{Nr}$ has an accelerating influence on fluid flow. Physically, it is due to the fact that an increase in the radiation parameter $\mathrm{Nr}$ for fixed values of other parameters the fluid velocity increases. From figure 5, it is seen that the velocity increases with increasing values of Volume fraction nano particle $\phi$. Figure 6 illustrates the influences of Prandtl number Pr on the velocity profiles. It is evident from the figure that the fluid velocity is increasing the plate velocity in the regions of close boundary. The effects of $\mathrm{Q}$ on velocity distribution is represented in figure 7. From this figure it is observed that the velocity decreases as an increase in heat source source parameter.

Figure 8 illustrates the influence of Prandtl number Pr on the fluid temperature. It is inferred that the thickness of thermal boundary layer is greatest at 0.68 . Also an increase in the prandtl number results in decrease of temperature. Pr signifies the relative effects of viscosity to thermal conductivity. The reason is that smaller values of Prandtl number are equivalent to increasing thermal conductivity and therefore heat is able to diffuse away from the heated surface more rapidly than for higher than for higher values of prandtl. In figure 9 the evolution of dimensionless temperature profiles $\theta(\mathrm{y}, \mathrm{t})$ inside the boundary layer, against span wise coordinate y for different values of BoltzmannRosseland radiation parameter $\mathrm{Nr}$. $\mathrm{Nr}$ corresponds to an increase in relative contribution of thermal radiation heat transfer to thermal conduction heat transfer. From this figure it is depicted that an increase in radiation parameter leads to an increase in the temperature in the boundary layer region which implies that radiation tends to enhance fluid temperature.

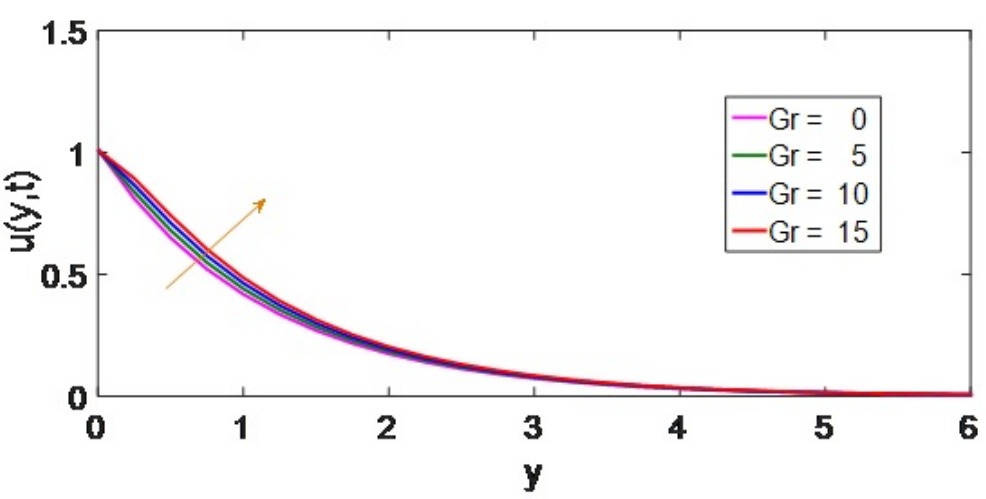

Fig. 2 Velocity profiles for Grashof number (Gr)

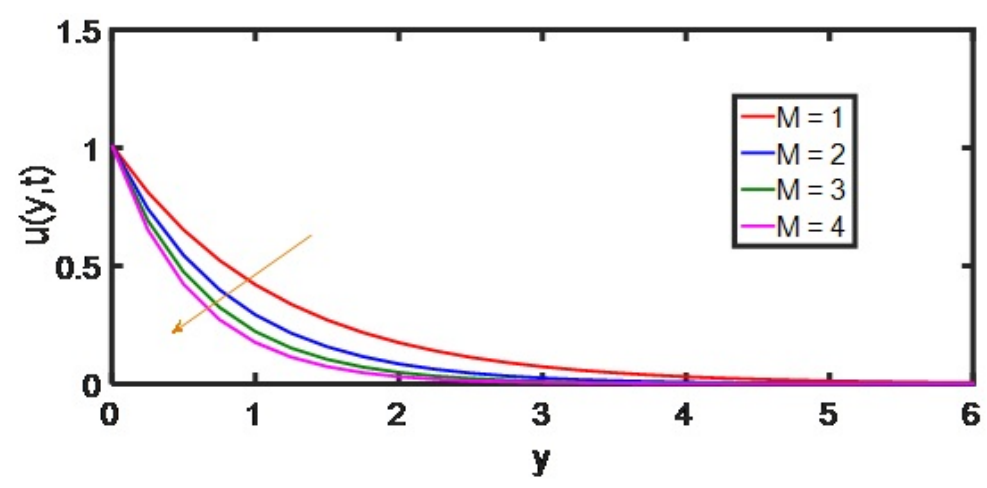

Fig. 3 Velocity profiles for Magnetic strength parameter (M)

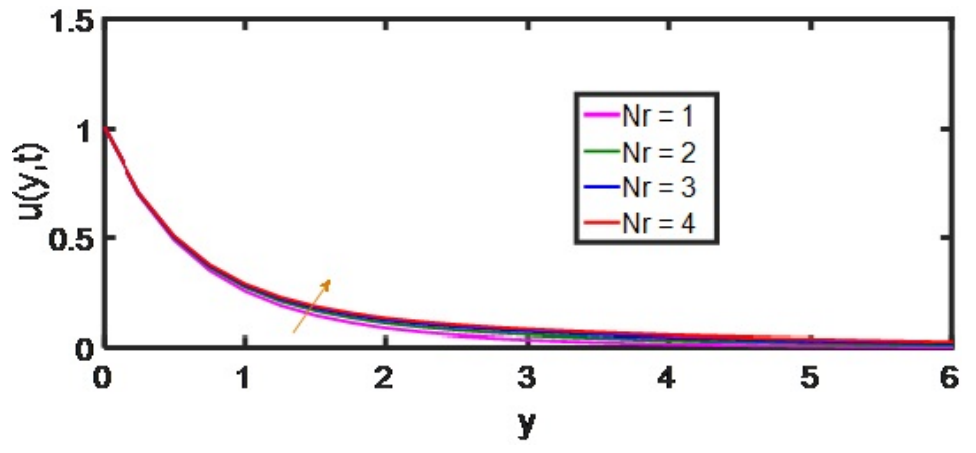

Fig. 4 Velocity profiles for Radiation parameter $(\mathrm{Nr})$

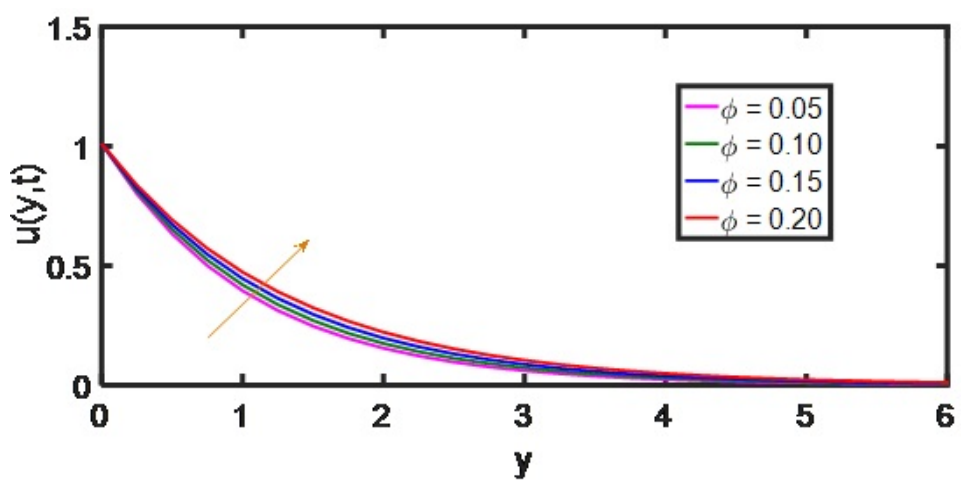

Fig. 5 Velocity profiles for Volume fraction nano particle $(\phi)$. 


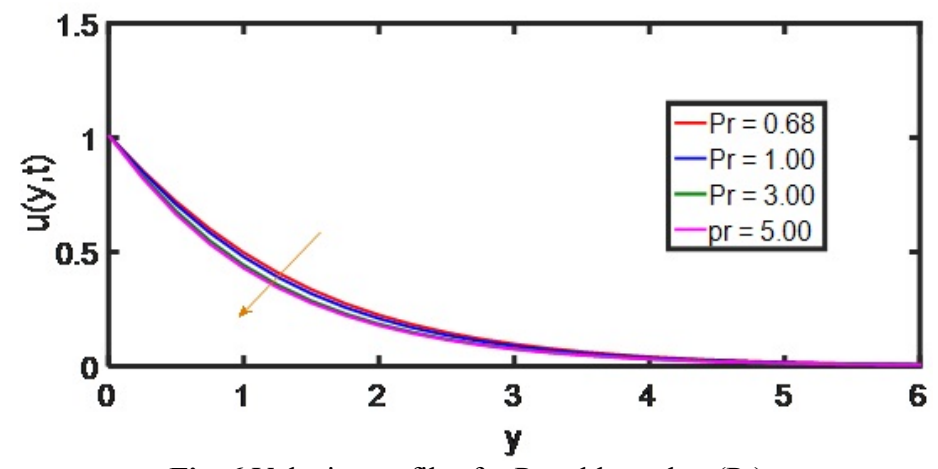

Fig. 6 Velocity profiles for Prandtl number (Pr).

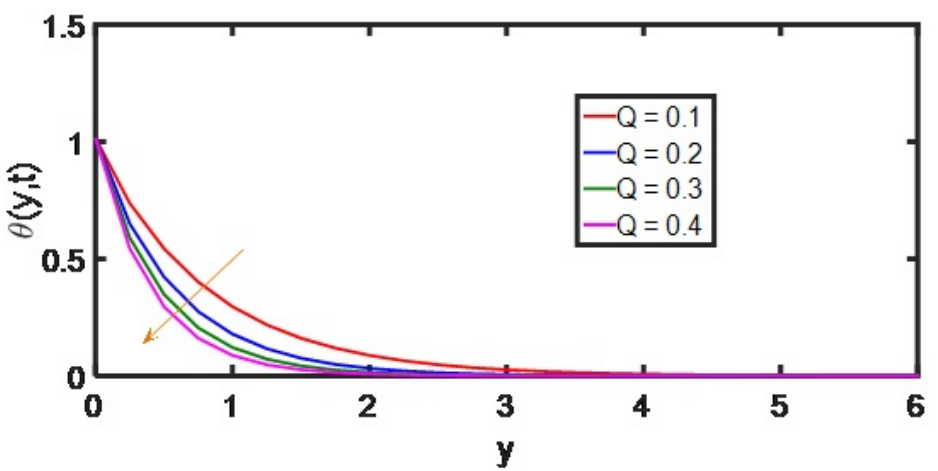

Fig. 7 Temperature profiles for heat source parameter (Q).

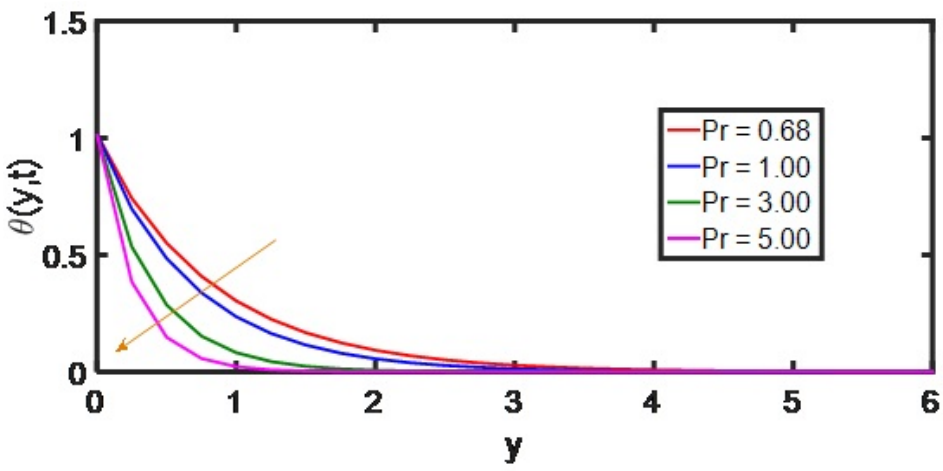

Fig. 8 Temperature profiles for Prandtl number (Pr)

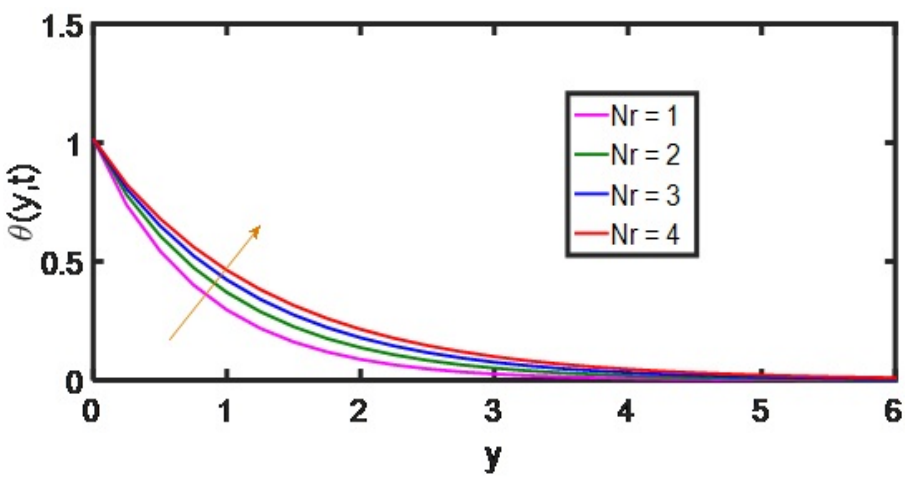

Fig. 9 Temperature profiles for Radiation parameter(Nr)

The numerical values of the skin-friction coefficient and Nusselt number for the nanoparticle $\mathrm{TiO}_{2}$ are presented in Table 2 and 3. From Table 2, it is seen that the local skin-friction coefficient increases with the increasing values of $\phi, \mathrm{Gr}$ and $\mathrm{Nr}$. On the other side $\mathrm{C}_{\mathrm{f}}$ decreases with the increasing values of $\mathrm{M}, \operatorname{Pr}$ and $\mathrm{Q}$. from Table 3, it is clear that the local Nusselt number decreases with increasing values of $\mathrm{Nr}$ and increases with Q and Pr.

Table 2: Numerical values of Skin-friction coefficient $\left(\mathrm{C}_{\mathrm{f}}\right)$

\begin{tabular}{|c|c|c|c|c|c|c|}
\hline$\Phi$ & GR & M & NR & PR & Q & CF \\
\hline 0.05 & & & & & & -0.9364 \\
0.15 & & & & & & -0.8172 \\
0.25 & & & & & & -0.7009 \\
0.35 & & & & & & -0.5879 \\
\hline & 1 & & & & & -0.8765 \\
& 2 & & & & & -0.8547 \\
& 3 & & & & & -0.8329 \\
& 4 & & & & & -0.8111 \\
\hline & & 2 & & & & -1.2429 \\
& & 3 & & & & -1.5244 \\
& & 4 & & & & -1.7617 \\
& & 5 & & & & -1.9709 \\
\hline & & & 2 & & & -0.8725 \\
& & & 3 & & & -0.8693 \\
& & & 4 & & & -0.8666 \\
& & & 5 & & & -0.8643 \\
\hline & & & & 0.68 & & -0.8466 \\
& & & & 1.00 & & -0.8528 \\
& & & & 3.00 & & -0.8678 \\
& & & & 5.00 & & -0.8735 \\
\hline & & & & & 0.2 & -0.8821 \\
& & & & & 0.3 & -0.8848 \\
& & & & & 0.5 & -0.8864 \\
& & & & & -0.8876 \\
\hline
\end{tabular}

Table 3: Numerical values of Nusselt number

\begin{tabular}{|c|c|c|c|}
\hline $\mathrm{Q}$ & $\mathrm{PR}$ & $\mathrm{NR}$ & $\mathrm{NU}$ \\
\hline 0.2 & & & 3.7028 \\
0.3 & & & 4.0903 \\
0.4 & & & 4.4169 \\
0.5 & & & 4.7046 \\
\hline & 0.68 & & 3.1296 \\
& 0.69 & & 3.1526 \\
& 0.70 & & 3.1753 \\
& 0.71 & & 3.1979 \\
\hline & & 2 & 2.6111 \\
& & 3 & 2.2613 \\
& & 4 & 2.0225 \\
& & 5 & 1.8463 \\
\hline
\end{tabular}

\section{CONCLUSIONS}

The present paper systematically examinations impacts of the thermal radiation and the heat source on the transient free convection flow of fluids streaming over a vertical infinite isothermal plate subject to vertical uniform magnetic field. Analytical solutions speaking to the flow and temperature fields are generated for the considered nanofluid, from which the wall shear and heat transfer rate are determined. The results of the present research are as per the following:

$>$ The temperature profiles are reduced by increasing absorption, otherwise an effect is present by heat generation. The thermal radiation further raises the temperature.

$>$ The rate of heat transfer is enhanced for heat absorption, whereas it reduced for heat generation and thermal radiation

$>$ The nusselt number and skin friction coefficient are shown to attain their steady states. 
$>$ The motion is considerably retarded when the vertical uniform magnetic field is active.

\section{NOMENCLATURE}

$\phi \quad$-nanoparticle volume fraction

$\mathrm{t} \quad$-scaled time

$\mathrm{t}^{*} \quad$-dimensionless time

$\sigma \quad$-electrical conductivity $\left(\mathrm{Sm}^{-1}\right)$

$\sigma^{*} \quad$-Stefan-Boltzmann constant $\left(\mathrm{Wm}^{-2} \mathrm{~K}^{-4}\right)$

$\rho \quad$-density $\left(\mathrm{kgm}^{-3}\right)$

$v \quad$-kinematic viscosity $\left(\mathrm{m}^{2} \mathrm{~s}^{-1}\right)$

$\mu \quad$-dynamic viscosity $\left(\mathrm{kgm}^{-1} \mathrm{~s}^{-1}\right)$

$\theta \quad$-scaled temperature

y -scaled coordinate

$\beta \quad$-thermal expansion coefficient $\left(\mathrm{K}^{-1}\right)$

$(\mathrm{x}, \mathrm{y}) \quad$-dimensional coordinate axes $(\mathrm{m})$

$\mathrm{u} 0 \quad$-characterstic velocity $\left(\mathrm{ms}^{-1}\right)$

$\mathrm{u} \quad$-dimensional velocity component $\left(\mathrm{ms}^{-1}\right)$

$\mathrm{U} \quad$-nondimensional velocity component

$\mathrm{T} \quad$-temperature of the fluid(K)

$\mathrm{q}_{\mathrm{w}} \quad$-heat flux $\left(\mathrm{Wm}^{-2}\right)$

$\mathrm{qr}_{\mathrm{r}} \quad$-heat flux by radiation $\left(\mathrm{Wm}^{-2}\right)$

$\mathrm{Q}_{0} \quad$-dimensional heat source $\left(\mathrm{Wm}^{-3}\right)$

Q -dimensionless heat source parameter

Pr -Prandtl number

$\mathrm{Nr} \quad$-radiation parameter

$\mathrm{Nu} \quad$-Nusselt number

M -magnetic strength parameter

$\mathrm{k} \quad$-thermal conductivity $\left(\mathrm{Wm}^{-1} \mathrm{~K}^{-1}\right)$

g -gravitational acceleration $\left(\mathrm{ms}^{-2}\right)$

$\mathrm{Cp} \quad$-specfic heat at constant pressure $\left(\mathrm{Jkg}^{-1} \mathrm{~K}^{-1}\right)$

$\mathrm{C}_{\mathrm{f}} \quad$-skinfriction coefficient

$\mathrm{B}_{0} \quad$-magnetic field $\left(\mathrm{Am}^{-1}\right)$

\section{Subscripts}

$\begin{array}{ll}\mathrm{f} & \text {-fluid property } \\ \infty & \text {-ambient value } \\ \mathrm{w} & \text {-wall value } \\ \mathrm{s} & \text {-nanoparticle property } \\ \mathrm{nf} & \text {-nanofluid }\end{array}$

\section{REFERENCES}

Akbarzadeh, M., Rashidi, S., Bovand, R., and Ellahi, R., 2016, “ A Sensitivity Analysis on Thermal and Pumping Power for the Flow of Nanofluid Inside a Wavy Channel", Journal of Molecular Liquids, 220, $1-13$.

http://dx.doi.org/ 10.1016/j.molliq.2016.04.058

Arpaci, V. S., 1987, "Radiative Entropy Production- Lost Heat into Entropy", International Journal of Heat and Mass Transfer, 30, 21152123.

http://dx.doi.org/10.1016/0017-9310(87)90090-1

Babu, M.J., Sandeep, N. and Raju, C.S.K., 2016, "Heat and Mass Transfer in MHD Eyring-Powell Nanofluid Flow Due To Cone in Porous Medium," International Journal of Engineering Research in Africa, 19, 57-74.

http://dx.doi.org/10.4028/www.scientific.net/JERA.19.57

Bejan,A., 1982, "Second-Law Analysis in Heat Transfer and Thermal Design", Advances in Heat Transfer, 15, 1-58.

http://dx.doi.org/10.1016/S0065-2717(08)70172-2
Buongiorno, J., 2006, "Convective Transport in Nanofluids". ASME Journal of Heat Transfer, 128, 240-250.

http://dx.doi.org/10.1115/1.2150834

Choi, S. U. S., Zhang, Z. G., Yu, W., Lockwood, F. E., Grulke, E. A., 2001, "Anomalous Thermal Conductivity Enhancement in Nanotube Suspensions," Applied Physics Letters, 79, 2252.

http://dx.doi.org/10.1063/1.1408272

Hamad, M.A.A., 2011, "Analytical Solution of Natural Convection Flow of a Nanofluid Over a Linearly Stretching Sheet in the Presence of Magnetic Field," International Communications in Heat and Mass Transfer, 38, 487-492.

http://dx.doi.org/10.1016/j.icheatmasstransfer.2010.12.042

Ibrahim, W., Makinde, O.D., 2015, "Magnetohydrodynamic Stagnation Point Flow and Heat Transfer of Casson Nanofluid Past a Stretching Sheet with Slip and Convective Boundary Condition," Journal of Aerospace Engineering 29, 04015037. https://doi.org/10.1061/(ASCE)AS.1943-5525.0000529

Kakac, S., and Pramunjaroenkij, A., 2009, "Review of convective heat transfer enhancement with nanofluids", Int. J. Heat Mass Transfer, 52(13), 3187-3196. https://doi.org/10.1016/j.ijheatmasstransfer.2009.02.006

Magyari, E., and Pantokratoras, A. (2011), "Note on the effect of thermal radiation in the linearized Rosseland approximation on the heat transfer Characteristics of various boundary layer flows," Int. Commun. Heat Mass Transfer, 38(5), 554-556.

http://dx.doi.org/10.1016/j.icheatmasstransfer.2011.03.006

Makhatar N.A.M, Saleh, H. and Ishak, H., 2015, "Flow Reversal of Fully Developed Mixed Convection of Nanofluids in a Vertical Channel Filled with Porous Medium," International Journal of Pure and Applied Mathematics, 103(1), 81-97. http://dx.doi.org/10.12732/ijpam.v103i1.7

Makinde, O.D., 2005, "Free Convection Flow with Thermal Radiation and Mass Transfer Past a Moving Vertical Porous Plate", Int. Comm. in Heat and Mass Transfer, 32 (10), 1411-1419.

http://dx.doi.org/10.1016/j.icheatmasstransfer.2005.07.005

Njane, W.N.M., Makinde, O.D. 2013, "Combined Effects of Buoyancy Force and Navier Slip on Magnetohydrodynaic Flow of a Nanofluid Over a Convectively Heated Vertical Porous Plate," The Sci.W.J. 2013(2013)8 pages.

http://dx.doi.org/10.115/2013/72643.

Nasrin, R., Alim, M.A., Chamkha, A.J., 2012, "Buoyancy-Driven Heat Transfer of Water-A12O3 Nanofluid in a Closed Chamber: Effects of Solid Volume Fraction, Prandlt Number and Aspect Ratio," Int.J. Heat and MassTransfer.55, 7355-7365.

https://doi.org/10.1016/j.ijheatmasstransfer.2012.08.011

Raju, C.S.K. and Sandeep, N., 2016, "Heat and Mass Transfer in MHD Non-Newtonian Bio-Convection Flow over a Rotating Cone/Plate with Cross Diffusion," Journal of Molecular Liquids, 215, 115-126. http://dx.doi.org/10.1016/i.molliq.2015.12.058

Raju, C.S.K., Sandeep, N. and Malvandi, A., 2016, "Free Convective Heat and Mass Transfer of MHD Non-Newtonian Nanofluids over a Cone in the Presence of Non-Uniform Heat Source/Sink," J. Molecular Liquids, 221, 101-115. http://dx.doi.org/10.1016/j.molliq.2016.05.078 
Raju, C.S.K., Sandeep, N. and Sugunamma, V., 2016, "Dual Solutions For Three-Dimensional MHD Flow Of A Nanofluid Over A Nonlinearly Permeable Stretching Sheet," Alexandria Engineering Journal, 55, 151-162.

http://dx.doi.org/10.1016/j.aej.2015.12.017

Ramesh, G.K., Chamkha, A.J., and Gireesha, B.J., 2016, "Boundary Layer Flow Past an Inclined Stationary/Moving Flat Plate with Convective Boundary Condition", Afrika Matematika, 27, 87-95.

https://doi.org/10.1007/s13370-015-0323-x

Satya Narayana, P. V., Venkateswarlu, B., 2016, "Heat and Mass Transfer on MHD Nanofluid Flow Past a Vertical Porous Plate in a Rotating System," Journal of Frontiers in Heat and Mass Transfer, 7, $1-10$.

https://doi.org/10.5098/hmt.7.8

Sandeep, N., Sulochana, C. and Animasaun, I.L., 2016, "StagnationPoint Flow of a Jeffrey Nano Fluid over a Stretching Surface with Induced Magnetic Field and Chemical Reaction," Int. J. Eng. Research in Afrika, 20, 93-111.

http://dx.doi.org/10.4028/www.scientific.net/JERA.20.93

Tiwari, R.K., and Das, M.K.,2007, "Heat transfer augment in a twosided lid-driven differently heated square cavity utilizing nanofluids", Int.J.Heat Mass Transfer,50(9),2002-2018.

doi:10.1016/j.ijheatmasstransfer.2006.09.034

Turkyilmazoglu, M., 2013, "unsteady convection flow of some nanofluids past a moving vertical flat plate with heat trasfer", J.Heat Transfer, 136(3), 031704-031707.

https://doi.org/10.1115/1.4025730
Wang, X.Q., Mujumdar, A. S., 2008, “A Review of Nanofluids - Part 2; Experiments and Applications", Brazilian Journal of Chemical Engineering, 25, 631-648.

http://dx.doi.org/10.1590/S0104-66322008000400002.

Woods, L.C., 1975, "Thermodynamics of fluid Systems", Oxford niversity Press, Oxford.

Xie, H. W. Y., Wei, C., 2010, "MgO Nanofluids: Higher Thermal Conductivity and Lower Viscosity among Ethylene Glycol-based Nanofluids Containing Oxide Nanoparticles," Journal of Experimental Nanoscience, 5(5), 463-472. http://dx.doi.org/10.1080/17458081003628949

Yue, H., Zhao, Y., Ma, X., Gong, J., 2012, "Ethylene Glycol: Properties, Synthesis, and Applications," Chemical Society Reviews, 41(11), 4218.

http://dx.doi.org/10.1039/c2cs15359a

Zhang, Y. B., 2016, "The Flow Equation for a Nanoscale Fluid Flow," International Journal of Heat and Mass Transfer, 92, 1004-1008. https://doi.org/10.1016/j.ijheatmasstransfer.2015.09.008

Zhang, Y. B., 2017, "Influence of pore wall surface property on flux of nanoporous filtering membrane," Frontiers in Heat and Mass Transfer, 9, 26, 1-6.

https://DOI: $10.5098 / \mathrm{hmt} .9 .26$

Zhang, Y. B., 2018, "A tree-type cylindrical-shaped nanoporous filtering membrane," Frontiers in Heat and Mass Transfer, 10, 16, 1-5. https://DOI: $10.5098 / \mathrm{hmt} .10 .16$ 